IFUP-TH 15/95

LMU-TPW 94-14

q-alg/9505023

\title{
VECTOR FIELDS ON QUANTUM GROUPS
}

\author{
Paolo Aschieri ${ }^{1}$ and Peter Schupp ${ }^{2}$ \\ ${ }^{1}$ Scuola Normale Superiore \\ Piazza dei Cavalieri 7, 56100 Pisa, Italy \\ and \\ Istituto Nazionale di Fisica Nucleare, sezione di Pisa, Italy \\ ${ }^{2}$ Sektion Physik der Ludwig-Maximilians-Universität München \\ Theoretische Physik - Lehrstuhl Professor Wess \\ Theresienstr. 37, D-80333 München
}

\begin{abstract}
We construct the space of vector fields on a generic quantum group. Its elements are products of elements of the quantum group itself with left invariant vector fields. We study the duality between vector fields and 1-forms and generalize the construction to tensor fields. A Lie derivative along any (also non left invariant) vector field is proposed and a puzzling ambiguity in its definition discussed. These results hold for a generic Hopf algebra.
\end{abstract}

e-mail: Aschieri@ux2sns.sns.it, Schupp@lswes8.ls-wess.physik.uni-muenchen.de 


\section{Introduction}

Following the program of generalizing the differential geometry structures to the non-commutative case, we construct on a Hopf algebra the analog of the space of vector fields.

Indeed in the literature the quantum Lie algebra of left invariant vector fields as well as the space of 1 -forms has been extensively analyzed [2, 3, 13, 4, 16, 8, 7], while the notion of generic vector field on a Hopf algebra and the duality relation with the space of 1-forms deserves more study [9, 10].

We will see how left invariant vector fields generate the whole space of vector fields. This space can be also characterized as the bicovariant bimodule (vector bundle) dual to that of 1 -forms.

Throughout this paper we will deal with a Hopf algebra $A$ [11] over $\boldsymbol{C}$ with coproduct $\Delta: A \rightarrow A \otimes A$, counit $\varepsilon: A \rightarrow C$ and invertible antipode $S: A \rightarrow A$. Particular cases of Hopf algebras are quantum groups, which for us will be Hopf algebras with one (or more) continuous parameter $q$; when $q=1$ the product "." in $A$ becomes commutative and we obtain the algebra of functions on a group. When we will speak of commutative case we will refer to the Hopf algebra $C^{\infty}(G)$ of smooth functions on a (compact) Lie group $G$.

In Section 2 we briefly recall how to associate a Connes-type differential calculus to a given Hopf algebra and we emphasize the role played by the tangent vectors. This construction will be effected along the lines of Woronowicz' work [2]. Indeed the results in [2] apply also to a general Hopf algebra with invertible antipode (not necessary a compact matrix pseudogroup). This can be shown by checking that all the formulae used for the construction - collected in the appendix of [2] - hold also in the case of a Hopf algebra with invertible antipode $\square$.

While following the work of [2] in spirit, we however decided on a small but important change of conventions 13] in this presentation. It is based on the following observation: while not a priori obvious, it is indeed possible to write all defining relations of the differential calculus as deformed commutation relations. Now, given that this is possible, we would like to have operators acting from the left and to the right as one is used to. This made the change in conventions necessary. Convertation to the old conventions (denoted by a "W" subscript) is possible in two ways: either one substitutes

$$
\chi_{i}=-S^{-1}\left({ }_{W} \chi_{i}\right), \quad f_{j}^{i}=S^{-1}\left({ }_{W} f_{j}^{i}\right), \quad \text { etc. }
$$

into our equations as will be mentioned in the text, or one simply reads all equations "from the left to the right". Notice that the ad-invariant right ideal $R$, the differential and the forms are the same. For quick reference we would like to point out the first version [12] of this article, where the old convention was used.

\footnotetext{
${ }^{1}$ Formula (A.22) in [2] is the most difficult to prove and necessitates the further axiom of the invertibility of the antipode $S$; also the invertibility of the map $s$ in (A.18) relies on the existence of $S^{-1}$. All the other formulae are direct consequences of the Hopf algebra axioms.
} 
In Section 3 we construct the space of vector fields, while in Section 4 we study the action of the Hopf algebra on the vector fields; i.e. we will study the pushforward of vector fields on Hopf algebras. Then we deal with (covariant and contravariant) tensor fields and wedge products.

In the last three sections we introduce and discuss Lie derivatives and a contraction operator on differential forms along generic vector fields. These two operators are basic tools for the formulation of deformed gravity theories [5], where the relevant Lie algebra is the $q$-Poincaré Lie algebra.

\section{Differential Geometry on Hopf Algebras}

In the commutative case, given the differential calculus on a (compact) Lie group $G$, we can consider the subspace in the space of all smooth functions $f: G \longrightarrow C$ defined by:

$$
R \equiv\left\{h \in C^{\infty}(G) \mid h\left(1_{G}\right)=0 \text { and } d h\left(1_{G}\right)=0\right\},
$$

where $1_{G}$ is the unit of the group.

$R$ is a particular ideal of the Hopf algebra $C^{\infty}(G)$ namely $(\operatorname{ker} \epsilon)^{2}$. All the information about the differential calculus on $G$ is contained in $R$.

Indeed the space of tangent vectors at the origin of the group is given by all the linear functionals which annihilate $R$ and any constant function. Locally we write a basis as $\left\{\left.\partial_{i}\right|_{1_{G}}\right\}$. Once we have this basis, using the tangent map (namely $T L_{g}$ ) induced by the left multiplication of the group on itself: $L_{g} g^{\prime}=g g^{\prime}, \forall g, g^{\prime} \in G$ we can construct a basis of left invariant vector fields $\left\{t_{i}\right\}$. The action of these vector fields on a generic function $a$ on the group manifold is (here and in the following: $\Delta(a) \equiv a_{1} \otimes a_{2}$ is the coproduct in Sweedler's notation [11])

$$
t_{i}(a)=\left.a_{1}\left(\left.\partial_{i} a_{2}\right|_{1_{G}}\right) \equiv \partial_{i}\right|_{1_{G}} * a
$$

in compliance with the following picture:

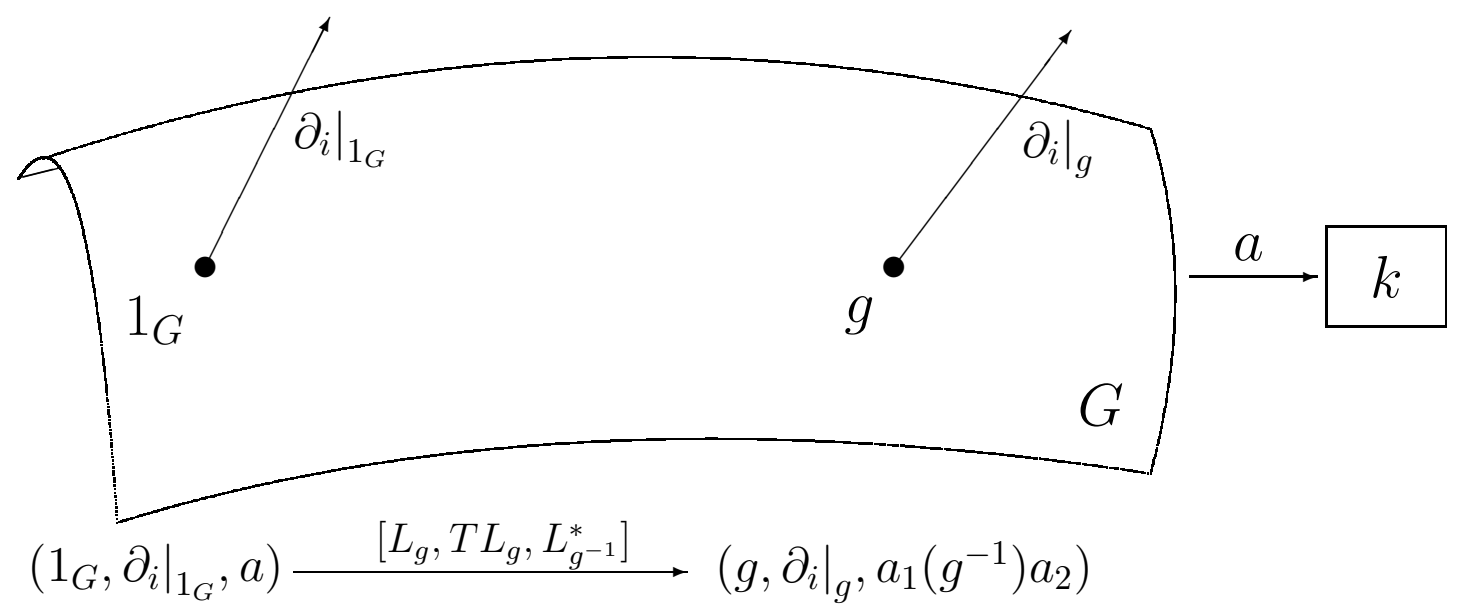

Note that $L_{g}^{*}(a)(h):=a(g h)=a_{1}(g) a_{2}(h)$. 
A generic 1-form can be written $\rho=\omega^{i} f_{i}\left[f_{i} \in C^{\infty}(G)\right]$ where $\left\{\omega^{i}\right\}$ is the dual basis of $\left\{t_{i}\right\}$. Finally, the differential on functions is

$$
d=\omega^{i} t_{i} \quad \text { that is } \quad d f=\omega^{i} t_{i}(f) .
$$

In [2] the quantum analog of $R \subset A$ is studied. Similarly to the classical case $R$ is a right ideal of $A$, formally $R A \subset R$, it is Ad-invariant, i.e. $\forall r \in R: r_{2} \otimes S\left(r_{1}\right) r_{3} \in$ $R \otimes A$, and its elements have vanishing counit. Given a Hopf algebra $A$, it turns out that we can always find an $R$ and construct a differential calculus (in general not unique).

The space of tangent vectors on $A$ is then defined as:[

$$
T \equiv\{\chi: A \rightarrow C \mid \chi \text { linear functionals, } \chi(I)=0 \text { and } \chi(S R)=0\},
$$

where $I$ is the unit of $A$ (in the commutative case it is the constant function $I(g)=1$ $\forall g \in G)$. In the sequel $\{I\}$ is the linear span of $I$. Let $\left\{\chi_{i}\right\} i=1, \ldots, n$ be a basis of $T$. Consider the linear space $X$ such that

$$
A=X \oplus R \oplus\{I\}
$$

$X$ is maximal in the (ordered) set of all linear subspaces of $A$ disjoint from $R \oplus\{I\}$. From (2.3) it follows that the dual vector space $X^{*}$ is isomorphic to $T$ and therefore there are $n$ elements $x^{i} \in X \subset$ ker $\varepsilon$ uniquely defined by the duality

$$
\chi_{i}\left(x^{j}\right)=\delta_{i}^{j} .
$$

Note that $\varepsilon\left(x^{i}\right)=0$ since $X \subset \operatorname{ker} \varepsilon$ because $A=\operatorname{ker} \varepsilon \oplus\{I\}$. We can then define the $n^{2}$ linear functionals $f_{j}{ }^{i}: A \longrightarrow C$

$$
\forall a \in A \quad f_{j}^{i}(a) \equiv \chi_{j}\left(a x^{i}\right)
$$

and (2.4)] and we have (from $A S(R)=S(R)$ )

$$
\chi_{i}(a b)=\chi_{i}(a) \varepsilon(b)+f_{i}{ }^{j}(a) \chi_{j}(b) .
$$

This is the deformed Leibniz rule for the operators $\chi_{i}$. In the $q=1$ case, when $R$ becomes the set defined in (2.1), we have $\chi_{i}=\left.\partial_{i}\right|_{1_{G}}, f_{j}{ }^{i}=\delta_{j}^{i} \varepsilon$ and we write (2.6) as $\left.\partial_{i}(f h)\right|_{1_{G}}=\left(\left.\partial_{i} f\right|_{1_{G}}\right) h\left(1_{G}\right)+f\left(1_{G}\right)\left(\left.\partial_{i} h\right|_{1_{G}}\right)$.

For consistency with (2.6) the $f_{j}{ }^{i}$ must satisfy the conditions:

$$
\begin{aligned}
& f_{j}{ }^{i}(a b)=f_{j}{ }^{k}(a) f_{k}{ }^{i}(b) \\
& f_{j}{ }^{i}(I)=\delta_{j}^{i} .
\end{aligned}
$$

\footnotetext{
${ }^{2}$ There are two tangent spaces:
}

The one we have chosen and the one corresponding to $\chi(R)=0$ [2].

${ }^{3}$ Relation to the conventions of [2] (denoted by a "W" subscript): $\chi_{i}=-S^{-1}{ }_{W} \chi_{i}, \quad f_{j}{ }^{i}=S^{-1}{ }_{W} f^{i}{ }_{j}, \quad R={ }_{W} R, \quad x^{i}=-S_{W} x^{i}$. 
The space of left invariant vector fields ${ }_{i n v} \Xi$ is easily constructed from $T$. Using the coproduct $\Delta$ we define - as in the commutative case - $\chi * a=(i d \otimes \chi) \Delta(a) \equiv$ $a_{1} \chi\left(a_{2}\right)$ and

$$
{ }_{i n v} \Xi \equiv\{t \mid t=\chi * \text { where } \chi \in T\}
$$

There is a one to one correspondence $\chi_{i} \leftrightarrow t_{i}=\chi_{i} *$. In order to obtain $\chi_{i}$ from $\chi_{i} *$ we simply apply $\varepsilon$ :

$$
\left(\varepsilon \circ t_{i}\right)(a)=\varepsilon\left(a_{1}\right) \chi_{i}\left(a_{2}\right)=\chi_{i}(a),
$$

where we have used the Hopf algebra axioms.

${ }_{i n v} \Xi$ is the vector subspace of all linear maps from $A$ to $A$ that is isomorphic to $T$.

We have chosen this perspective to introduce the space of left invariant vector fields in order to point out that (also in the case of a general Hopf algebra) it has an existence on its own, independent of the space of 1-forms.

The space of 1 -forms $\Gamma$ is formed by all the elements $\rho$ that are written as formal products and sums of the type

$$
\rho=\omega^{i} a_{i}
$$

Here $a_{i} \in A$ and $\omega^{i} i=1, \ldots, n$ is the basis dual to $\left\{t_{i}\right\}$. We express this duality with a bracket:

$$
\left\langle\chi_{j}, \omega^{i}\right\rangle=\delta_{j}^{i}
$$

Relation (2.11) tells us that the space of 1-forms is freely generated by the elements $\omega^{i}$. By definition any $\rho$ is decomposable in a unique way as $\rho=\omega^{i} a_{i}$ and $\Gamma$ is a right $A$-module with the trivial product $\left(\omega^{i} a_{i}\right) b \equiv \omega^{i}\left(a_{i} b\right) . \Gamma$ is also a left $A$-module with the following left product:

$$
\forall b \in A \quad b \omega^{i}=\omega^{j}\left(f_{j}{ }^{i} * b\right) \equiv \omega^{j}\left(i d \otimes f_{j}^{i}\right) \Delta(b) .
$$

From this relation it follows that:

$$
\forall a \in A \quad \omega^{i} a=\left[\left(f_{j}{ }^{i} \circ S\right) * a\right] \omega^{j}=a_{1} f_{j}{ }^{i}\left(S a_{2}\right) \omega^{j}
$$

and that any $\rho$ can be written in a unique way in the form

$$
\rho=b_{i} \omega^{i}
$$

with $b_{i} \in A$.

Finally, the differential operator $d: A \longrightarrow \Gamma$ can be defined through the relation:

$$
\forall a \in A \quad d a=\omega^{i}\left(\chi_{i} * a\right) .
$$

Notice that this can be rewritten:

$$
d a=\omega^{i}\left(\chi_{i} * a\right)=S f_{i}^{j} *\left(\chi_{j} * a\right) \omega^{i}=\left(-S \chi_{i} * a\right) \omega^{i}
$$


where we have used (2.14) and (2.6), i.e. ${ }_{w} \chi_{i}=-S \chi_{i}$. As a consequence, the differential calculus obtained has the following properties [2]:

i) The differential operator satisfies the Leibniz rule

$$
d(a b)=(d a) b+a(d b) \quad \forall a, b \in A .
$$

Moreover any $\rho \in \Gamma$ can be expressed as

$$
\rho=d a_{\alpha} b_{\alpha}
$$

for some $a_{\alpha}, b_{\alpha}$ belonging to $A$. (Use $\omega^{i}=d x_{2}^{i} S^{-1} x_{1}^{i}$ below).

Remark. Once we know the operator $d$, the space of tangent vectors on $A$, like in the commutative case, can be defined as:

$$
T=\{\chi \mid \chi(a)=0 \text { if and only if } P d a=0\}
$$

where $P d a \equiv d a_{2} S^{-1} a_{1}$ with $\Delta(a)=a_{1} \otimes a_{2}$. The linear map $P$ is a projection operator; to a given form $\rho=\omega^{i} a_{i}$ it associates the form $P(\rho)=\varepsilon\left(a_{i}\right) \omega^{i}$ and in particular $P\left(d x^{i}\right)=\omega^{i}$. In the commutative case $\varepsilon\left(a_{i}\right)$ is the value that $a_{i} \in A=C^{\infty}(G)$ takes in the origin $1_{G}$ of the Lie group $G . P(\rho)$ is then the left invariant 1 -form whose value in the origin $1_{G}$ of the Lie group equals the value of the 1 -form $\rho$ in $1_{G}$. f $^{\text {f }}$

ii) The differential calculus is called bicovariant because using $d$ and the coproduct $\Delta$ we can define two linear compatible maps $\Delta_{\Gamma}$ and $\Gamma_{\Gamma} \Delta$

$$
\begin{aligned}
& \Delta_{\Gamma}(d a)=(i d \otimes d) \Delta(a), \quad \Delta_{\Gamma}: \Gamma \rightarrow A \otimes \Gamma \quad \text { (left covariance) } \\
& \Gamma_{\Gamma} \Delta(d a)=(d \otimes i d) \Delta(a), \quad{ }_{\Gamma} \Delta: \Gamma \rightarrow \Gamma \otimes A \quad \text { (right covariance) } \\
& \Delta_{\Gamma}(a \rho b)=\Delta(a) \Delta_{\Gamma}(\rho) \Delta(b), \quad{ }_{\Gamma} \Delta(a \rho b)=\Delta(a)_{\Gamma} \Delta(\rho) \Delta(b)
\end{aligned}
$$

which represent the left and right action of the Hopf algebra $A$ on $\Gamma$. In the commutative case they express the pull-back on 1-forms induced by the left or right multiplication of the group on itself [0]. $\Delta_{\Gamma}$ and ${ }_{\Gamma} \Delta$ are compatible in the sense that $\left(i d \otimes_{\Gamma} \Delta\right) \Delta_{\Gamma}=\left(\Delta_{\Gamma} \otimes i d\right)_{\Gamma} \Delta$. In the commutative case this formula tells us that the left and right actions of the group on $\Gamma$ commute: $R_{g}^{*} L_{g^{\prime}}^{*}=L_{g^{\prime}}^{*} R_{g}^{*} \forall g, g^{\prime} \in G$. From the definitions (2.21) and (2.22) one deduces the following properties [2]:

$$
\begin{aligned}
(\varepsilon \otimes i d) \Delta_{\Gamma}(\rho)=\rho, & (i d \otimes \varepsilon)_{\Gamma} \Delta(\rho)=\rho \\
(\Delta \otimes i d) \Delta_{\Gamma}=\left(i d \otimes \Delta_{\Gamma}\right) \Delta_{\Gamma}, & (i d \otimes \Delta)_{\Gamma} \Delta=\left({ }_{\Gamma} \Delta \otimes i d\right)_{\Gamma} \Delta .
\end{aligned}
$$

An element $\omega$ of $\Gamma$ is said to be left invariant if

$$
\Delta_{\Gamma}(\omega)=I \otimes \omega
$$

\footnotetext{
${ }^{4}$ In [2]: ${ }_{W} P d a=S\left(a_{1}\right) d a_{2}$, such that $\omega^{i}=P d x^{i}={ }_{W} P d_{W} x^{i}={ }_{W} \omega^{i}$.
} 
and right invariant if

$$
{ }_{\Gamma} \Delta(\omega)=\omega \otimes I
$$

We have seen that any $\rho$ is of the form $\rho=\omega^{i} a_{i}$. We have that the $\omega^{i}$ are left invariant and form a basis of ${ }_{i n v} \Gamma$, the linear subspace of all left invariant elements of $\Gamma$. Relation (2.12) tells us that ${ }_{i n v} \Gamma$ and ${ }_{i n v} \Xi$ are dual vector spaces.

iii) There exists an adjoint representation $M_{j}{ }^{i}$ of the Hopf algebra, defined by the right action on the $\omega^{i}$ :

$$
{ }_{\Gamma} \Delta\left(\omega^{i}\right)=\omega^{j} \otimes M_{j}{ }^{i} ; \quad M_{j}{ }^{i} \in A .
$$

The co-structures on the $M_{j}{ }^{i}$ can be deduced [2]:

$$
\begin{aligned}
& \Delta\left(M_{j}{ }^{i}\right)=M_{j}{ }^{l} \otimes M_{l}{ }^{i} \\
& \varepsilon\left(M_{j}{ }^{i}\right)=\delta_{j}^{i} \\
& S\left(M_{i}{ }^{j}\right)=\left(M^{-1}\right)_{i}{ }^{j}
\end{aligned}
$$

The elements $M_{j}{ }^{i}$ can be used to build a right invariant basis of $\Gamma$. Indeed the $\eta^{i}$ defined by

$$
\eta^{i} \equiv \omega^{j} S\left(M_{j}{ }^{i}\right)
$$

are a basis of $\Gamma$ (every element of $\Gamma$ can be uniquely written as $\rho=\eta^{i} b_{i}$ ) and their right invariance can be checked directly .

Moreover, from (2.31), using (2.32) and (2.14) one can prove the relation

$$
\left(a * f_{i}^{j}\right) M_{j}{ }^{k}=M_{i}{ }^{j}\left(f_{j}{ }^{k} * a\right)
$$

with $a * f_{i}{ }^{j} \equiv\left(f_{i}{ }^{j} \otimes i d\right) \Delta(a)$ being the action of the right-invariant operator $f_{i}{ }^{j}$ on the function $a$.

\section{Construction of the space of Vector Fields.}

In this section we study the space $\Xi$ of vector fields over Hopf algebras defining a left product between elements of $A$ and of ${ }_{i n v} \Xi$.

A generic vector field can be written in the form $f^{i} \cdot t_{i}$ where $\left\{t_{i}\right\} i=1, \ldots, n$ is a basis of left invariant vector fields and $f^{i}$ are $n$ smooth functions on the group manifold. In the commutative case $f^{i} \cdot t_{i}=t_{i} \square f^{i}$ i.e. left and right products (that we have denoted with $\square)$ are the same, indeed $\left(t_{i}\right.$ 口 $\left.f^{i}\right)(h) \equiv t_{i}(h) f^{i}=f^{i} t_{i}(h)=$ $\left(f^{i} \cdot t_{i}\right)(h)$.

Let $t_{i}=\chi_{i} *$ be a basis in ${ }_{i n v} \Xi$ and let $a^{i}, i=1, \ldots, n$ be generic elements of $A$ :

\section{Definition}

$$
\Xi \equiv\left\{V \mid V: A \longrightarrow A ; V=a^{i} \cdot t_{i}\right\}
$$


where the definition of the left product · is given below:

\section{Definition}

$$
\forall a, b \in A, \forall t \in{ }_{i n v} \Xi \quad(a \cdot t) b \equiv a t(b)=a(\chi * b) .
$$

The product - has a natural generalization to the whole $\Xi$ :

$$
\begin{aligned}
\cdot: \quad A \times \Xi & \longrightarrow \\
(a, V) & \longmapsto
\end{aligned} \quad \text { a } \quad \text { where } \quad \forall b \in A \quad(a \cdot V)(b) \equiv a V(b) .
$$

It is easy to prove that $(\Xi, \cdot)$ is a left $A$-module:

$$
(a+b) \cdot V=a \cdot V+b \cdot V ; \quad(a b) \cdot V=a \cdot(b \cdot V) ; \quad(\lambda a) \cdot V=\lambda a \cdot V
$$

with $\lambda \in \boldsymbol{C}$.

Note: The left product was chosen in the definition of $\Xi$ so that the symbol . can henceforth be omitted in all formulae. For the right product $\square$ — that we will introduce later — we $d o$ have to distinguish for instance the elements $V \square(a b) \in \Xi$ and $V(a b) \in A$ because the vector fields act to the right (by convention).

$\Xi$ is the analog of the space of derivations on the ring $C^{\infty}(G)$ of the smooth functions on the group $G$. Indeed we have:

$$
\begin{array}{ll}
V(a+b)=V(a)+V(b), \quad V(\lambda a)=\lambda V(a) & \text { Linearity } \\
V(a b) \equiv\left(c^{i} t_{i}\right)(a b)=V(a) b+c^{i}\left(f_{i}{ }^{j} * a\right) t_{j}(b) & \text { Leibniz rule }
\end{array}
$$

in the classical case $c^{i}\left(f_{i}{ }^{j} * a\right) t_{j}(b)=a V(b) \quad\left(\right.$ recall $\left.f_{i}{ }^{j}=\delta_{i}^{j} \varepsilon ; \varepsilon * b=b\right)$.

This last equation can be written as a commutation relation

$$
V a=V(a)+c^{i}\left(f_{i}^{j} * a\right) t_{j}
$$

and in a basis-independent version that uses the right product $\square(4.12)$ Ð

$$
V a=V(a)+V_{\square} a .
$$

We have seen the duality between ${ }_{i n v} \Gamma$ and ${ }_{i n v} \Xi$. We now extend it to $\Gamma$ and $\Xi$, where $\Gamma$ is seen as a right $A$-module (not necessarily a bimodule) and $\Xi$ is our left $A$-module.

Theorem 1. There exists a unique map

$$
\langle,\rangle: \Xi \times \Gamma \longrightarrow A
$$

\footnotetext{
${ }^{5}$ There is another basis-independent version of this equation based on a left $U$-coaction ${ }_{U} \Delta(V) \equiv V^{1^{\prime}} \otimes V^{2}$ (that generalizes the coproduct of $\left.{ }_{i n v} \Xi\right): \quad V a=V^{1^{\prime}}(a) V^{2}$.
} 
such that:

1) $\forall V \in \Xi$; the application

$$
\langle V,\rangle: \Gamma \longrightarrow A
$$

is a right $A$-module morphism, i.e. is linear and $\langle V, \rho a\rangle=\langle V, \rho\rangle a$.

2) $\forall \rho \in \Gamma$; the application

$$
\langle, \rho\rangle: \Xi \longrightarrow A
$$

is a left $A$-module morphism, i.e. is linear and $\langle b V, \rho\rangle=b\langle V, \rho\rangle$.

3) Given $\rho \in \Gamma$

$$
\langle, \rho\rangle=0 \Rightarrow \rho=0
$$

where $\langle, \rho\rangle=0$ means $\langle V, \rho\rangle=0 \quad \forall V \in \Xi$.

4) Given $V \in \Xi$

$$
\langle V,\rangle=0 \Rightarrow V=0
$$

where $\langle V\rangle=$,0 means $\langle V, \rho\rangle=0 \quad \forall \rho \in \Gamma$.

5) On ${ }_{i n v} \Xi \times_{i n v} \Gamma$ the bracket $\langle$,$\rangle acts as the one introduced in the previous$ section.

Remark. Properties 3) and 4) state that $\Gamma$ and $\Xi$ are dual $A$-moduli, in the sense that they are dual with respect to $A$.

Proof

Properties 1), 2) and 5) uniquely characterize this map . To prove the existence of such a map we show that the following bracket

\section{Definition}

$$
\langle V, \rho\rangle \equiv V\left(a_{\alpha}\right) b_{\alpha},
$$

where $a_{\alpha}, b_{\alpha}$ are elements of $A$ such that $\rho=d a_{\alpha} b_{\alpha}$, satisfies 1),2) and 5).

We first verify that the above definition is well given, that is:

$$
\text { Let } \rho=d a_{\alpha} b_{\alpha}=d a_{\beta}^{\prime} b_{\beta}^{\prime} \text { then } V\left(a_{\alpha}\right) b_{\alpha}=V\left(a_{\beta}^{\prime}\right) b_{\beta}^{\prime} .
$$

Indeed, since

$$
d a_{\alpha} b_{\alpha}=d a_{\beta}^{\prime} b_{\beta}^{\prime} \Leftrightarrow \omega^{i} t_{i}\left(a_{\alpha}\right) b_{\alpha}=\omega^{i} t_{i}\left(a_{\beta}^{\prime}\right) b_{\beta}^{\prime} \Leftrightarrow t_{i}\left(a_{\alpha}\right) b_{\alpha}=t_{i}\left(a_{\beta}^{\prime}\right) b_{\beta}^{\prime}
$$

[we used the uniqueness of the decomposition (2.11]), the definition is consistent because

$$
V\left(a_{\alpha}\right) b_{\alpha}=V\left(a_{\beta}^{\prime}\right) b_{\beta}^{\prime} \Leftrightarrow c^{i} t_{i}\left(a_{\alpha}\right) b_{\alpha}=c^{i} t_{i}\left(a_{\beta}^{\prime}\right) b_{\beta}^{\prime}
$$

where $V=c^{i} t_{i}$. 
Property 1) is trivial since $\rho a=\left(d a_{\alpha} b_{\alpha}\right) a=d a_{\alpha}\left(b_{\alpha} a\right)$.

Property 2) holds since

$$
\langle c V, \rho\rangle=(c V)\left(a_{\alpha}\right) b_{\alpha}=c\left(V\left(a_{\alpha}\right) b_{\alpha}\right)=c\langle V, \rho\rangle .
$$

Property 5). Let $\left\{\omega^{i}\right\}$ and $\left\{t_{i}\right\}$ be dual bases in ${ }_{i n v} \Gamma$ and ${ }_{i n v} \Xi$. Since $\omega^{i} \in \Gamma, \omega^{i}=$ $d a_{\alpha} b_{\alpha}$ for some $a_{\alpha}$ and $b_{\alpha}$ in $A$. We can also write $\omega^{i}=d a_{\alpha} b_{\alpha}=\omega^{k} t_{k}\left(a_{\alpha}\right) b_{\alpha}$, so that, due to the uniqueness of the decomposition (2.11), we have

$$
t_{k}\left(a_{\alpha}\right) b_{\alpha}=\delta_{k}^{i} I \quad(I \operatorname{unit} \text { of } A)
$$

we then obtain

$$
\left\langle t_{j}, \omega^{i}\right\rangle=t_{j}\left(a_{\alpha}\right) b_{\alpha}=\delta_{j}^{i} I
$$

Property 3). Let $\rho=\omega^{i} a_{i} \in \Gamma$.

If $\langle V, \rho\rangle=0 \quad \forall V \in \Xi$, in particular $\left\langle t_{j}, \rho\right\rangle=0 \quad \forall j=1, \ldots, n$; then $\left\langle t_{j}, \omega^{i}\right\rangle a_{i}=0 \Leftrightarrow$ $a_{j}=0$, and therefore $\rho=0$.

Property 4). Let $V=a^{i} t_{i} \in \Xi$.

If $\langle V, \rho\rangle=0 \quad \forall \rho \in \Gamma$, in particular $\left\langle V, \omega^{j}\right\rangle=0 \quad \forall j=1, \ldots, n$; then $a^{i}\left\langle t_{i}, \omega^{j}\right\rangle=0 \Leftrightarrow$ $a^{j}=0$, and therefore $V=0$.

By construction every $V$ is of the form

$$
V=a^{i} t_{i}
$$

We can now show the unicity of such a decomposition.

Theorem 2. Any $V \in \Xi$ can be uniquely written in the form

$$
V=a^{i} t_{i}
$$

Proof

Let $V=a^{i} t_{i}=a^{i} t_{i}$ then

$$
\forall i=1, \ldots, n \quad a^{i}=a^{j}\left\langle t_{j}, \omega^{i}\right\rangle=\left\langle V, \omega^{i}\right\rangle=a^{\prime j}\left\langle t_{j}, \omega^{i}\right\rangle=a^{i i}
$$

Notice that once we know the decomposition of $\rho$ and $V$ in terms of $\omega^{i}$ and $t_{i}$, the evaluation of $\langle$,$\rangle is trivial:$

$$
\langle V, \rho\rangle=\left\langle b^{j} t_{j}, \omega^{i} a_{i}\right\rangle=b^{j}\left\langle t_{j}, \omega^{i}\right\rangle a_{i}=b^{i} a_{i} .
$$

Vice versa from the previous theorem $V=\left\langle V, \omega^{i}\right\rangle t_{i}$ and $\rho=\omega_{i}\left\langle t_{i}, \rho\right\rangle$. 
We conclude this section summarizing the three different ways of looking at $\Xi$ :

(I) $\quad \Xi$ as the set of all deformed derivations over $A$ [see (3.1), (3.5) - (3.8)].

(II) $\quad \Xi$ as the left $A$-module freely generated by the elements $t_{i}, \quad i=1, \ldots, n$. The latter is the set of all the formal products and sums of the type $a^{i} t_{i}$, where $a^{i}$ are generic elements of $A$. Indeed, by virtue of Theorem 2, the map that associates to each $V=a^{i} \cdot t_{i}$ in $\Xi$ the corresponding element $a^{i} t_{i}$ is an isomorphism between left $A$-moduli.

(III) $\quad \Xi$ as $\Xi^{\prime}=\{U: \Gamma \longrightarrow A, U$ linear and $U(\rho a)=U(\rho) a \forall a \in A\}$, i.e. $\Xi$ as the dual (with respect to $A$ ) of the space of 1 -forms $\Gamma$. The space $\Xi^{\prime}$ has a trivial left $A$-module structure: $(a U)(\rho) \equiv a U(\rho) . \Xi$ and $\Xi^{\prime}$ are isomorphic left $A$ moduli because of property (3.10) which states that to each $\langle V, \quad\rangle: \Gamma \rightarrow A$ there corresponds one and only one $V \cdot\left[\langle V\rangle=,\left\langle V^{\prime},\right\rangle \Rightarrow V=V^{\prime}\right]$. Every $U \in \Xi^{\prime}$ is of the form $U=\langle V, \quad\rangle$; more precisely, if $a^{i}$ is such that $U\left(\omega^{i}\right)=a^{i}$ then $U=\left\langle a^{i} t_{i}, \quad\right\rangle$.

These three ways of looking at $\Xi$ will correspond to different aspects of the Cartan Calculus: the Lie derivatives $\ell_{V}$ will generalize (I), inner derivations $i_{V}$ will correspond to (III), while the transformation properties of $\ell_{V}$ and $i_{V}$ are governed by (II).

\section{Bicovariant Bimodule Structure.}

In [2] the space of 1 -forms is extensively studied. A right and a left product are introduced between elements of $\Gamma$ and of $A$, and it is known how to obtain a right product from a left one [e.g. $\left.a \omega^{i}=\omega^{j}\left(f_{j}{ }^{i} * a\right), \omega^{i} a=\left(S f_{j}{ }^{i} * a\right) \omega^{j}\right]$, i.e. $\Gamma$ is a bimodule over $A$.

Since the actions $\Delta_{\Gamma}$ and ${ }_{\Gamma} \Delta$ are compatible in the sense that:

$$
\left(i d \otimes_{\Gamma} \Delta\right) \Delta_{\Gamma}=\left(\Delta_{\Gamma} \otimes i d\right)_{\Gamma} \Delta
$$

the bimodule $\Gamma$ is called a bicovariant bimodule. In [2] it is shown that relations (2.7), (2.8), (2.14), (2.33), (2.29), (2.30) and (2.28) completely characterize the bicovariant bimodule $\Gamma$.

In Section 3 we have studied the left product - and we have seen that $\Xi$ is a left bimodule over $A$ [see (3.4)]. In this section we introduce a right product and a left and right action of the Hopf algebra $A$ on $\Xi$. The left and right actions $\Delta_{\Xi}$ and $\Xi \Delta$ are the $q$-analog of the push-forward of tensor fields on a group manifold. Similarly to $\Gamma$ also $\Xi$ is a bicovariant bimodule.

The construction of the right product on $\Xi$, of the right action $\Xi \Delta$ and of the left action $\Delta_{\Xi}$ will be effected along the lines of Woronowicz' Theorem 2.5 in [2], whose statement can be explained in the following steps:

Theorem 3. Consider the symbols $t_{i}(i=1, \ldots, n)$ and let $\Xi$ be the left $A$-module freely generated by them: 


$$
\Xi \equiv\left\{a^{i} t_{i} \mid a^{i} \in A\right\}
$$

Consider functionals $O_{i}{ }^{j}: A \longrightarrow C$ satisfying [see (2.7) and (2.8)]

$$
\begin{aligned}
& O_{i}{ }^{j}(a b)=O_{i}{ }^{k}(a) O_{k}{ }^{j}(b) \\
& O_{i}{ }^{j}(I)=\delta_{j}^{i}
\end{aligned} .
$$

Introduce a right product via the definition [see (2.14)]

\section{Definition}

$$
\left(a^{i} t_{i}\right) \square b \equiv a^{i}\left(O_{i}{ }^{j} * b\right) t_{j}
$$

It is easy to prove that

i) $\Xi$ is a bimodule over $A$. (A proof of this first statement as well as of the following ones is contained in [2]).

Introduce a left action (push-forward) of the Hopf algebra $A$ on $\Xi$

\section{Definition}

$$
\Delta_{\Xi}\left(a^{i} t_{i}\right) \equiv \Delta\left(a^{i}\right)\left(I \otimes t_{i}\right)
$$

It follows that

ii) $\quad\left(\Xi, \Delta_{\Xi}\right)$ is a left covariant bimodule over $A$, that is

$$
\Delta_{\Xi}(a V b)=\Delta(a) \Delta_{\Xi}(V) \Delta(b) ; \quad(\varepsilon \otimes i d) \Delta_{\Xi}(V)=V ; \quad(\Delta \otimes i d) \Delta_{\Xi}=\left(i d \otimes \Delta_{\Xi}\right) \Delta_{\Xi} .
$$

Introduce $n^{2}$ elements of $A, N^{i}{ }_{j}$, satisfying [see (2.33), (2.29) and (2.30)]

$$
\begin{gathered}
N^{i}{ }_{j}\left(a * O_{i}{ }^{k}\right)=\left(O_{j}{ }^{i} * a\right) N^{k}{ }_{i} \\
\Delta\left(N^{j}{ }_{i}\right)=N^{j}{ }_{l} \otimes N^{l}{ }_{i} \\
\varepsilon\left(N^{j}{ }_{i}\right)=\delta_{j}^{i},
\end{gathered}
$$

and introduce a right action $\Xi \Delta$ such that [see (2.28)]

\section{Definition}

$$
{ }_{\Xi} \Delta\left(a^{i} t_{i}\right) \equiv \Delta\left(a^{i}\right)\left(t_{j} \otimes N^{j}{ }_{i}\right)
$$

Then it can be proven that

iii) The elements [see (2.32)]

$$
h_{i} \equiv S^{-1}\left(N^{j}{ }_{i}\right) t_{j}
$$

are right invariant: $\Xi \Delta\left(h_{i}\right)=h_{i} \otimes I$. Moreover any $V \in \Xi$ can be expressed in a unique way respectively as $V=a^{i} h_{i}$ and as $V=h_{i} b^{i}$, where $a^{i}, b^{i} \in A$. 
iv) $(\Xi, \Xi \Delta)$ is a right covariant bimodule over $A$, that is ${ }_{\Xi} \Delta(a V b)=\Delta(a)_{\Xi} \Delta(V) \Delta(b) ; \quad(i d \otimes \varepsilon)_{\Xi} \Delta(V)=V ; \quad(i d \otimes \Delta)_{\Xi} \Delta=\left({ }_{\Xi} \Delta \otimes i d\right)_{\Xi} \Delta$.

v) The left and right covariant bimodule $\left(\Xi, \Delta_{\Xi}, \Xi \Delta\right)$ is a bicovariant bimodule, that is left and right actions are compatible:

$$
\left(i d \otimes{ }_{\Xi} \Delta\right) \Delta_{\Xi}=\left(\Delta_{\Xi} \otimes i d\right)_{\Xi} \Delta
$$

In the previous section we have seen [remark (II)] that the space of vector fields $\Xi$ is the free left $A$-module generated by the symbols $t_{i}$, so that the above theorem applies to our case.

There are many bimodule structures (i.e. choices of $O_{i}^{j}$ ) $\Xi$ can be endowed with. Using the fact that $\Xi$ is dual to $\Gamma$ we request compatibility with the $\Gamma$ bimodule. In the commutative case $\left\langle f t_{j}, \omega^{i}\right\rangle=\left\langle t_{j} f, \omega^{i}\right\rangle=\left\langle t_{j}, f \omega^{i}\right\rangle=\left\langle t_{j}, \omega^{i} f\right\rangle$.

In the quantum case we know that $\left\langle t_{j}, \omega^{i} a\right\rangle=\left\langle a t_{j}, \omega^{i}\right\rangle$ and we require

$$
\left\langle t_{j} \square a, \omega^{i}\right\rangle=\left\langle t_{j}, a \omega^{i}\right\rangle ;
$$

this condition uniquely determines the bimodule structure of $\Xi$. Indeed we have

$$
t_{i} a=\left(f_{i}^{j} * a\right) t_{j}
$$

i.e.

$$
O_{i}{ }^{j} \equiv f_{i}{ }^{j}
$$

This is in fact the bimodule structure needed for the product rule (3.8). So far $\Xi$ has a bimodule structure. $\Xi$ becomes a left covariant bimodule if we define $\Delta_{\Xi}$ as in (4.5) so that $t_{i}$ are left invariant vector fields. The following consideration fixes the right covariance of $\Xi$ : For reasons of symmetry let us try to impose

$$
\left\langle t_{i}, \omega^{j}\right\rangle=\delta_{i}^{j}=\left\langle h_{i}, \eta^{j}\right\rangle
$$

where $t_{i}$ and $\omega^{j}$ are left-invariant and $h_{i}$ and $\eta^{j}$ are the canonically associated right-invariant objects; see (4.10) and (2.32). As can easily be seen this condition is equivalent to the following definition of the $n^{2}$ elements $N_{k}^{l} \in A$

\section{Definition}

$$
N_{k}^{l}=S\left(M_{k}^{l}\right)
$$

Theorem 4. The $N^{l}{ }_{k}$ elements defined above satisfy relations (4.8), (4.7) and (4.6):
1) $\varepsilon\left(N^{j}{ }_{i}\right)=\delta_{j}^{i}$
2) $\Delta\left(N^{j}{ }_{i}\right)=N^{j}{ }_{l} \otimes N^{l}{ }_{i}$
3) $N_{k}^{i}\left(a * O_{i}{ }^{j}\right)=\left(O_{k}{ }^{i} * a\right) N^{j}{ }_{i}$ 
Proof

1) is trivial.

2) use $N^{i}{ }_{j}=S M_{j}{ }^{i}$ and $\Delta \circ S=\sigma \circ(S \otimes S) \circ \Delta$, where $\sigma_{A}$ is the flip map in $A \otimes A$.

3) Using $N^{i}{ }_{j}=S M_{j}{ }^{i}$ and $O_{i}{ }^{j}=f_{i}{ }^{j}$ in $(2.33$ ) gives

$$
\left(a * O_{k}^{i}\right) S^{-1} N_{i}^{j}=S^{-1} N_{k}^{i}\left(O_{i}{ }^{j} * a\right) .
$$

Multiplying with " $N$ " on both sides we obtain relation 3).

In equations (2.2) and (2.16) elements of $\Xi$ and $\Gamma$ make a joint appearance. To be still able to talk about transformation properties of such expressions we need to combine the previously introduced actions into one object, $\Delta_{A}$, simply by putting $\Delta_{A} \equiv{ }_{\Xi} \Delta$ on $\Xi$ and $\Delta_{A} \equiv{ }_{\Gamma} \Delta$ on $\Gamma$ and requiring $\Delta_{A}$ to be an algebra homomorphism. From this definition we get the following important corollary:

Corollary. The expression $\omega^{i} t_{i}$ in (2.2) is invariant in the sense that

$$
\Delta_{A}\left(\omega^{i} t_{i}\right)={ }_{\Gamma} \Delta\left(\omega^{i}\right)_{\Xi} \Delta\left(t_{i}\right)=\omega^{j} t_{k} \otimes M_{j}{ }^{i} N^{k}{ }_{i}=\omega^{i} t_{i} \otimes 1
$$

Similar statements apply to ${ }_{A} \Delta$.

The left-invariant vector fields were characterized in (2.9) through their left action $t_{i}(a)=\chi_{i} * a$ on functions. It would be nice if a similar equation were true for the right-invariant vector fields $h_{i}$; indeed we have

Theorem 5. $\quad h_{i}(a) \equiv S^{-1}\left(N^{j}{ }_{i}\right) t_{j}(a)=a * \chi_{i} \quad \forall a \in A$.

Proof

From (2.2) we have

$$
\omega_{a} \equiv P d a \equiv d\left(a_{2}\right) S^{-1} a_{1}=\omega^{i} \chi_{i}(a)
$$

and in particular $\omega_{x^{i}}=\omega^{i}$. Using this equation we can rewrite the definition of $M_{i}^{k},(2.28)$, as $M_{i}^{k}=\chi_{i}\left(x_{2}^{k}\right) x_{3}^{k} S^{-1} x_{1}^{k}$, or

$$
M_{i}^{j} \chi_{j}\left(x^{k}\right)=\chi_{i}\left(x_{2}^{k}\right) x_{3}^{k} S^{-1} x_{1}^{k}
$$

We now show that this equation is still true for an arbitrary function $a \in A$ in place of $x^{k}$. Indeed from (2.3) any function can be decomposed into a part that is spanned by the $x^{i}$ and a part that is contained in $R \oplus\{I\}$ i.e. is annihilated by the $\chi_{j}$. But $R$ and $\{I\}$ are Ad-invariant so the part of $a$ that is contained in $R \oplus\{I\}$ contributes to neither side of the equation in question and we have in fact:

$$
M_{i}^{j} \chi_{j}(a)=\chi_{i}\left(a_{2}\right) a_{3} S^{-1} a_{1}
$$


Definition (4.15) and a slight rearrangement (take $a=b_{2}$ and multiply by $b_{1}$ ) finally gives

$$
S^{-1} N^{j}{ }_{i} a_{1} \chi_{j}\left(a_{2}\right)=\chi_{i}\left(a_{1}\right) a_{2}
$$

and proves the theorem.

Remark. Statement 3) of Theorem 4 is in fact a consequence of Theorem 5. Both are a consequence of a more general Hopf algebra relation (e.g. eqn. 4.67 of [14]) that is valid in the semi-direct product algebra of $A$ and $A^{*}$.

Now that we have all the ingredients, the construction of the bicovariant bimodule $\Xi$ is easy and straightforward. For example $\Xi \Delta$ is given in formula (4.9).

We can then conclude that $\left(\Xi, \Delta_{\Xi}, \Xi \Delta\right)$ is a bicovariant bimodule.

Notice that, since Theorem 3 completely characterizes a bicovariant bimodule all the formulas containing the symbols $f_{i}{ }^{j}$ or $M_{k}{ }^{l}$ or elements of $\Gamma$ are still valid under the substitutions $f_{i}{ }^{j} \rightarrow S^{-1} O_{i}{ }^{j}, \quad M_{k}{ }^{l} \rightarrow N_{k}^{l}$ and $\Gamma \rightarrow \Xi$.

\section{Tensor fields}

The construction completed for vector fields is readily generalized to $p$-times contravariant tensor fields.

We define $\Xi \otimes \Xi$ to be the space of all elements that can be written as finite sums of the kind $\sum_{i} V_{i} \otimes V_{i}^{\prime}$ with $V_{i}, V_{i}^{\prime} \in \Xi$. The tensor product (in the algebra $A$ ) between $V_{i}$ and $V_{i}^{\prime}$ has the following properties:

$$
V \square a \otimes V^{\prime}=V \otimes a V^{\prime}, a\left(V \otimes V^{\prime}\right)=(a V) \otimes V^{\prime} \text { and }\left(V \otimes V^{\prime}\right) \square a=V \otimes\left(V^{\prime}{ }_{\square} a\right)
$$

so that $\Xi \otimes \Xi$ is naturally a bimodule over $A$.

Left and right actions on $\Xi \otimes \Xi$ are defined by:

$$
\begin{aligned}
& { }_{A} \Delta\left(V \otimes V^{\prime}\right) \equiv V_{1} V^{\prime}{ }_{1} \otimes V_{l} \otimes V^{\prime}{ }_{l}, \quad{ }_{A} \Delta: \Xi \otimes \Xi \rightarrow A \otimes \Xi \otimes \Xi \\
& \Delta_{A}\left(V \otimes V^{\prime}\right) \equiv V_{r} \otimes V^{\prime}{ }_{r} \otimes V_{1} V^{\prime}{ }_{1}, \quad \Delta_{A}: \Xi \otimes \Xi \rightarrow \Xi \otimes \Xi \otimes A
\end{aligned}
$$

where $V_{1} \otimes V_{l}$ and $V_{r} \otimes V_{1}$ are defined by

$$
\begin{aligned}
& \Delta_{\Xi}(V)=V_{1} \otimes V_{l} \quad \in A \otimes \Xi, \\
& \Xi \Delta(V)=V_{r} \otimes V_{1} \quad \in \Xi \otimes A .
\end{aligned}
$$

More generally, we can introduce the actions on $\Xi^{\otimes p} \equiv \underbrace{\Xi \otimes \Xi \otimes \cdots \otimes}_{p \text {-times }}$ as

$$
{ }_{A} \Delta\left(V \otimes V^{\prime} \otimes \cdots \otimes V^{\prime \prime}\right) \equiv V_{1} V^{\prime}{ }_{1} \cdots V^{\prime \prime}{ }_{1} \otimes V_{l} \otimes V^{\prime}{ }_{l} \otimes \cdots \otimes V^{\prime \prime}{ }_{l}
$$




$$
\begin{gathered}
{ }_{A} \Delta: \Xi^{\otimes p} \longrightarrow A \otimes \Xi^{\otimes p} ; \\
\Delta_{A}\left(V \otimes V^{\prime} \otimes \cdots \otimes V^{\prime \prime}\right) \\
\equiv V_{r} \otimes V^{\prime}{ }_{r} \otimes \cdots \otimes V^{\prime \prime}{ }_{r} \otimes V_{1} V^{\prime}{ }_{1} \cdots V^{\prime \prime}{ }_{1} \\
\Delta_{A}: \Xi^{\otimes p} \longrightarrow \Xi^{\otimes p} \otimes A .
\end{gathered}
$$

Left invariance on $\Xi \otimes \Xi$ is naturally defined as ${ }_{A} \Delta\left(V \otimes V^{\prime}\right)=I \otimes V \otimes V^{\prime}$ (similar definition for right invariance), so that for example $t_{i} \otimes t_{j}$ is left invariant, and is in fact a left invariant basis for $\Xi \otimes \Xi$ : each element can be written as $a^{i j} t_{i} \otimes t_{j}$ in a unique way.

It is not difficult to show that $\Xi \otimes \Xi$ is a bicovariant bimodule. In the same way also $\left(\Xi^{\otimes p},{ }_{A} \Delta, \Delta_{A}\right)$ is a bicovariant bimodule. An analog procedure, using $\Delta_{\Gamma}$ and $\Gamma_{\Gamma} \Delta$ instead of $\Delta_{\Xi}$ and $\Xi \Delta$, applies also to $\Gamma^{\otimes n}$ the $n$-times tensor product of 1 -forms.

Any element $v \in \Xi^{\otimes p}$ can be written as $v=b^{i_{1} \ldots i_{p}} t_{i_{1}} \otimes \ldots t_{i_{p}}$ in a unique way, similarly any element $\tau \in \Gamma^{\otimes n}$ can be written as $\tau=\omega^{i_{1}} \otimes \ldots \omega^{i_{n}} a_{i_{1} \ldots i_{n}}$ in a unique way. It is now possible to generalize the previous bracket $\langle\rangle:, \Xi \times \Gamma \rightarrow A$ to $\Xi^{\otimes p}$ and $\Gamma^{\otimes n}$. For $p>n:\langle v, \tau\rangle=0$; for $p \leq n$ :

$$
\begin{aligned}
& \langle,\rangle: \Xi^{\otimes p} \times \Gamma^{\otimes n} \longrightarrow \Gamma^{\otimes n-p} \\
& (v, \tau) \quad \longmapsto\langle v, \tau\rangle=b^{j_{1} \ldots j_{p}}\left\langle t_{j_{1}} \otimes \ldots t_{j_{p}}, \omega^{i_{1}} \otimes \ldots \omega^{i_{n}}\right\rangle a_{i_{1} \ldots i_{n}} \\
& =b^{i_{p} \ldots i_{1}} \omega^{i_{p+1}} \otimes \ldots \omega^{i_{n}} a_{i_{1} \ldots i_{n}}
\end{aligned}
$$

where $\Gamma^{\otimes 0} \equiv A, \Gamma^{\otimes 1} \equiv \Gamma$ and we have defined

$$
\begin{aligned}
\left\langle t_{j_{p}} \otimes \ldots t_{j_{1}}, \omega^{i_{1}} \otimes \ldots \omega^{i_{n}}\right\rangle & \equiv\left\langle t_{j_{p}} \otimes \ldots t_{j_{1}}, \omega^{i_{1}} \otimes \ldots \omega^{i_{p}}\right\rangle \omega^{i_{p+1}} \otimes \ldots \omega^{i_{n}} \\
& \equiv\left\langle t_{j_{1}}, \omega^{i_{1}}\right\rangle \ldots\left\langle t_{j_{p}}, \omega^{i_{p}}\right\rangle \omega^{i_{p+1}} \otimes \ldots \omega^{i_{n}} \\
& =\delta_{j_{1}}^{i_{1}} \ldots \delta_{j_{p}}^{i_{p}} \omega^{i_{p+1}} \otimes \ldots \omega^{i_{n}}
\end{aligned}
$$

(with the obvious convention for the special cases $p=n-1$ and $p=n$ ). Using definition (5.9) it is easy to prove that

$$
\left\langle v_{\square} a, \tau\right\rangle=\langle v, a \tau\rangle
$$

namely

$$
\begin{aligned}
\left\langle t_{j_{1}} \otimes \ldots t_{j_{p} \square} a, \omega^{i_{1}} \otimes \ldots \omega^{i_{n}}\right\rangle & =\left(f_{j_{1}}{ }^{k_{1}} * \ldots f_{j_{p}}{ }^{k_{p}} * a\right)\left\langle t_{k_{1}} \otimes \ldots t_{k_{p}}, \omega^{i_{1}} \otimes \ldots \omega^{i_{n}}\right\rangle \\
& =\left(f_{j_{1}}{ }^{i_{p}} * \ldots f_{j_{p}}{ }^{i_{1}} * a\right) \omega^{i_{p+1}} \otimes \ldots \omega^{i_{n}} \\
\left\langle t_{j_{1}} \otimes \ldots t_{j_{p}}, a \omega^{i_{1}} \otimes \ldots \omega^{i_{n}}\right\rangle & =\left\langle t_{j_{1}} \otimes \ldots t_{j_{p}}, \omega^{l_{1}} \otimes \ldots \omega^{l_{n}}\right\rangle\left(f_{l_{n}}{ }^{i_{n}} * \ldots f_{l_{1}}{ }^{i_{1}} * a\right) \\
& =\delta_{j_{p}}^{l_{1}} \ldots \delta_{j_{1}}^{l_{p}} \omega^{l_{p+1}} \otimes \ldots \omega^{l_{n}}\left(f_{l_{n}}^{i_{n}} * \ldots f_{l_{1}}{ }^{i_{1}} * a\right) \\
& =\left(f_{j_{1}}{ }^{i_{p}} * \ldots f_{j_{p}}{ }^{i_{1}} * a\right) \omega^{i_{p+1}} \otimes \ldots \omega^{i_{n}}
\end{aligned}
$$

and (5.10) is verified if and only if (5.9) holds. 
Therefore we have also shown that definition (5.9) is the only one compatible with property (5.10), i.e. property (5.10) uniquely determines the coupling between $\Xi^{\otimes}$ and $\Gamma^{\otimes}$.

It is also easy to prove that the bracket $\langle, \quad\rangle$ extends to $\Xi^{\otimes p}$ and $\Gamma^{\otimes p}$ the duality between $\Xi$ and $\Gamma$.

More generally we can define $\Xi^{\otimes} \equiv A \oplus \Xi \oplus \Xi^{\otimes 2} \oplus \Xi^{\otimes 3}$.. to be the algebra of contravariant tensor fields (and $\Gamma^{\otimes}$ that of covariant tensor fields).

The actions ${ }_{A} \Delta$ and $\Delta_{A}$ have a natural generalization to $\Xi^{\otimes}$ so that we can conclude that $\left(\Xi^{\otimes},{ }_{A} \Delta, \Delta_{A}\right)$ is a bicovariant graded algebra, the graded algebra of tensor fields over the ring "of functions on the group" $A$, with the left and right "push-forward" ${ }_{A} \Delta$ and $\Delta_{A}$.

Before we can introduce a Lie derivative and an inner derivation we need a wedge product of forms, i.e. we have to briefly discuss antisymmetrized covariant tensor fields.

\subsection{Bicovariant graded algebras}

A general method to construct new tensor fields starting from $\Gamma^{\otimes}$ is to quotient the bicovariant graded algebra $\Gamma^{\otimes}$ with a graded ideal $S$. In order for $\Gamma^{\otimes} / S$ to be bicovariant, the ideal $S$ has also to be biinvariant (i.e. a left and right subcomodule). For example the ideal can be generated by the elements $S_{i j}^{k l} \omega^{i} \otimes \omega^{j}$ 回 and a sufficient condition for the biinvariance is $S_{i j}^{k l} M_{k}{ }^{m} M_{l}{ }^{n}=M_{i}{ }^{k} M_{j}{ }^{l} S_{k l}^{m n}$, where $\Xi \Delta \omega^{i}=\omega^{j} \otimes M_{j}{ }^{i}$.

Particular cases of such a quotient procedure are when $S$ is the kernel of a graded bimodule homomorphism $A: \Gamma^{\otimes} \rightarrow \Gamma^{\otimes}$ then $\Gamma^{\otimes} / \operatorname{ker}(A)$ is isomorphic to the image of $A$ and therefore is a biinvariant graded subalgebra of $\Gamma^{\otimes}$. This is what we require for a wedge product construction. The wedge product of two forms is given by

$$
\begin{gathered}
\omega^{i} \wedge \omega^{j}=A\left(\omega^{i} \otimes \omega^{j}\right)=\omega^{k} \otimes \omega^{l} W_{k l}^{i j} \in \Gamma^{\wedge 2} \subset \Gamma^{\otimes 2} \\
a \omega^{i} \wedge \omega^{j} \equiv a\left(\omega^{i} \wedge \omega^{j}\right) ; \quad \omega^{i} \wedge \omega^{j} b \equiv\left(\omega^{i} \wedge \omega^{j}\right) b
\end{gathered}
$$

Since the homomorphism $A$ is required to commute with the coactions ${ }_{A} \Delta$ and $\Delta_{A}$ the new tensor transforms covariantly according to its index structure:

$$
\Delta_{A}\left(\omega^{i} \wedge \omega^{j}\right) \equiv \Delta_{A}\left(\omega^{k} \otimes \omega^{l}\right) W_{k l}^{i j}=\omega^{k} \wedge \omega^{l} \otimes M_{k}^{i} M_{l}^{j}
$$

thus imposing

$$
W_{i j}^{k l} M_{k}^{m} M_{l}^{n}=M_{i}^{k} M_{j}^{l} W_{k l}^{m n}
$$

on $W$ as a necessary condition. In short tensor product notation: $\omega_{1} \wedge \omega_{2}=$ $\omega_{1} \otimes \omega_{2} W_{12} \Rightarrow W_{12} M_{1} M_{2}=M_{1} M_{2} W_{12}$. Let $\hat{\sigma}_{i j}{ }^{k l}=f_{i}{ }^{l}\left(M_{j}{ }^{k}\right)$. Due to (2.33) any function $W(1, \hat{\sigma})$ will satisfy (5.13). We will in fact take $W=1-\hat{\sigma}$. For this choice and for examples of $n>2$ relations we would like to refer the reader to [2, 0, 9, 14];

\footnotetext{
${ }^{6}$ Or, what is the same, one imposes relations $S_{i j}^{k l} \omega^{i} \wedge \omega^{j}=0$.
} 
here we will just quote the results: generalizing equation (5.11), wedge products of $n$ forms are again expressed in terms of tensor fields:

$$
\omega_{1} \wedge \ldots \wedge \omega_{n}=\omega_{1} \otimes \ldots \otimes \omega_{n} W_{1 \ldots n}
$$

The numerical coefficients $W_{1 \ldots n}$ are given through a recursion relation

$$
W_{1 \ldots n}=W_{2 \ldots n} \mathcal{I}_{1 \ldots n}
$$

where

$$
\mathcal{I}_{1 \ldots n}=1-\hat{\sigma}_{12}+\hat{\sigma}_{12} \hat{\sigma}_{23}-\ldots-(-1)^{n} \hat{\sigma}_{12} \hat{\sigma}_{23} \cdots \hat{\sigma}_{n-1, n}
$$

and $W_{i}{ }^{j}=\mathcal{I}_{i}{ }^{j}=\delta_{i}^{j}$. As is easily seen, $\mathcal{I}$ has the following decomposition property that we will use in the next chapter:

$$
\mathcal{I}_{1 \ldots n}=\mathcal{I}_{1 \ldots k}+(-1)^{k} \hat{\sigma}_{12} \hat{\sigma}_{23} \cdots \hat{\sigma}_{k, k+1} \mathcal{I}_{k+1 \ldots n}
$$

The first order differential operator $d$ can be extended in a unique way to an exterior differential $d$ mapping $\mathrm{n}$-forms in $(\mathrm{n}+1)$-forms, satisfying the undeformed Leibniz rule and such that $d^{2}=0$ [2].

It is also straightforward to define antisymmetrized contravariant vector fields in this way.

\section{Contraction operator and Lie derivative}

In this last section we propose a definition of Lie derivative along a generic vector field. We start with the introduction of the contraction operator $i_{V}$, also called inner derivation, with $V \in \Xi$ based on equation (5.8).

For a generic vector field $V=b^{j} t_{j}$ we define:

\section{Definition}

$$
i_{V}(\vartheta)=\langle V, \vartheta\rangle \quad \vartheta \text { generic form. }
$$

Theorem 6 . The contraction operator satisfies the following properties: $\vartheta, \vartheta^{\prime}$ generic forms; $a, a_{i_{1} \ldots i_{n}} \in A ; V=b^{i} t_{i} ; \lambda \in \mathbf{C}$

a) $i_{V}(\vartheta)=i_{b^{j} t_{j}}(\vartheta)=b^{j} i_{t_{j}}(\vartheta)$

b) $i_{V}(a)=0$

c) $i_{V}\left(\omega^{j}\right)=b^{j}$

d) $i_{V}\left(\omega^{i_{1}} \wedge \ldots \omega^{i_{n}} a_{i_{1} \ldots i_{n}}\right)=i_{V}\left(\omega^{i_{1}} \wedge \ldots \omega^{i_{s}}\right) \wedge\left(\omega^{i_{s+1}} \wedge \ldots \omega^{i_{n}} a_{i_{1} \ldots i_{n}}\right)+$ $(-1)^{s} b^{i} f_{i}^{j} *\left(\omega^{i_{1}} \wedge \ldots \omega^{i_{s}}\right) \wedge i_{t_{j}}\left(\omega^{i_{s+1}} \wedge \ldots \omega^{i_{n}} a_{i_{1} \ldots i_{n}}\right)$ 
e) $i_{V}(a \vartheta)=b^{i}\left(f_{i}^{j} * a\right) i_{t_{j}}(\vartheta)$

f) $i_{V}\left(\vartheta a+\vartheta^{\prime}\right)=i_{V}(\vartheta) a+i_{V}\left(\vartheta^{\prime}\right)$

g) $i_{\lambda V}=\lambda i_{V}$

Proof.

Properties a), b), c), f), g) are direct consequences of (5.8), e) follows from (5.10). To proof $d$ ) we have to use the definition of the wedge product $(5.14-5.16)$ : first we note that (in tensor product notation)

$$
\begin{aligned}
i_{V}\left(\omega_{1} \wedge \ldots \wedge \omega_{n}\right) & =i_{V}\left(\omega_{1} \otimes \ldots \otimes \omega_{n} W_{1 \ldots n}\right) \\
& =i_{V}\left(\omega_{1}\right) \omega_{2} \otimes \ldots \otimes \omega_{n} W_{1 \ldots n} \\
& =i_{V}\left(\omega_{1}\right) \omega_{2} \wedge \ldots \wedge \omega_{n} \mathcal{I}_{1 \ldots n}
\end{aligned}
$$

where we have used (5.15) in the last step — in index notation:

$$
i_{t_{i}}\left(\omega^{i_{1}} \wedge \ldots \wedge \omega^{i_{n}}\right)=\omega^{j_{2}} \wedge \ldots \wedge \omega^{j_{n}} \mathcal{I}_{i j_{2} \ldots j_{n}}^{i_{1} \ldots i_{n}}
$$

next we can show

$$
\begin{aligned}
f_{i}^{j} *\left(\omega^{i_{1}} \wedge \ldots \wedge \omega^{i_{s}}\right) & =\omega^{k_{1}} \wedge \ldots \wedge \omega^{k_{s}} f_{i}^{j}\left(M_{k_{1}}{ }^{i_{1}} \ldots M_{k_{s}}{ }^{i_{s}}\right) \\
& =\omega^{k_{2}} \wedge \ldots \wedge \omega^{k_{s+1}} \hat{\sigma}_{i k_{2}}{ }^{i_{1} l_{2}} \hat{\sigma}_{l_{2} k_{3}}{ }^{i_{2} l_{3}} \ldots \hat{\sigma}_{l_{s} k_{s+1}}{ }^{i_{s} j}
\end{aligned}
$$

finally we utilize the decomposition property (5.17) and associativity of the wedge product (in tensor product notation)

$$
\begin{aligned}
i_{V}\left(\omega_{1} \wedge\right. & \left.\ldots \wedge \omega_{n}\right)= \\
=\quad & i_{V}\left(\omega_{1}\right) \omega_{2} \wedge \ldots \omega_{n} \mathcal{I}_{1 \ldots n} \\
=\quad & i_{V}\left(\omega_{1}\right)\left(\omega_{2} \wedge \ldots \omega_{s}\right) \wedge\left(\omega_{s+1} \wedge \ldots \omega_{n}\right)\left(\mathcal{I}_{1 \ldots s}+(-1)^{s} \hat{\sigma}_{12} \cdots \hat{\sigma}_{s, s+1} \mathcal{I}_{s+1 \ldots n}\right) \\
=\quad & i_{V}\left(\omega_{1} \wedge \ldots \wedge \omega_{s}\right) \wedge\left(\omega_{s+1} \wedge \ldots \wedge \omega_{n}\right) \\
& +b^{i} i_{t_{i}}\left(\omega_{1}\right)\left(\omega_{2} \wedge \ldots \omega_{s+1}\right) \wedge\left(\omega_{s+2} \wedge \ldots \omega_{n}(-1)^{s} \hat{\sigma}_{12} \ldots \hat{\sigma}_{s, s+1} \mathcal{I}_{s+1 \ldots n}\right) \\
=\quad & i_{V}\left(\omega_{1} \wedge \ldots \wedge \omega_{s}\right) \wedge \omega_{s+1} \wedge \ldots \wedge \omega_{n} \\
& +(-1)^{s} b^{i} f_{i}{ }^{j} *\left(\omega_{1} \wedge \ldots \wedge \omega_{s}\right) \wedge i_{t_{j}}\left(\omega_{s+1} \wedge \ldots \wedge \omega_{n}\right) .
\end{aligned}
$$

With property f) this proves $d$ ).

Remark 1. A slight generalization of property d) for two generic forms $\vartheta$ and $\vartheta^{\prime}$ is also true [use e)]:

$$
i_{V}\left(\vartheta \wedge \vartheta^{\prime}\right)=i_{V} \vartheta \wedge \vartheta^{\prime}+(-1)^{\operatorname{deg}(\vartheta)} b^{i} f_{i}^{j} * \vartheta \wedge i_{t_{j}} \vartheta^{\prime} .
$$


Remark 2. Property e) reduces in the commutative case to the familiar formula:

$$
i_{V}(h \vartheta)=h i_{V} \vartheta .
$$

The Lie derivative along left invariant vector fields is given by [7]:

$$
\ell_{t}(\tau) \equiv(i d \otimes \chi)_{\Gamma} \Delta(\tau)=\chi * \tau \quad \ell_{t}: \Gamma^{\otimes n} \longrightarrow \Gamma^{\otimes n}
$$

where $\chi \in{ }_{i n v} \Xi$ is such that $\chi^{*}=t$. On $\Gamma^{\wedge}$ it can be proven that [6, [0, [15] :

$$
\ell_{t_{i}}=i_{t_{i}} d+d i_{t_{i}}
$$

It is then natural to introduce the Lie derivative along a generic vector field $V$ through the following

\section{Definition}

$$
\ell_{V}=i_{V} d+d i_{V}
$$

Theorem 7. The Lie derivative satisfies the following properties:

1) $\ell_{V} a=V(a)$

2) $\ell_{V} d \vartheta=d \ell_{V} \vartheta$

3) $\ell_{V}\left(\lambda \vartheta+\vartheta^{\prime}\right)=\lambda \ell_{V} \vartheta+\ell_{V} \vartheta^{\prime}$

4) $\ell_{b V} \vartheta=b \ell_{V} \vartheta+d b \wedge i_{V} \vartheta$, where $\vartheta$ is a generic form

5) $\ell_{V}\left(\vartheta \wedge \vartheta^{\prime}\right)=\ell_{V} \vartheta \wedge \vartheta^{\prime}+b^{i}\left(f_{i}{ }^{j} * \vartheta\right) \wedge \ell_{t_{j}} \vartheta^{\prime}+(-1)^{\operatorname{deg}(\vartheta)} d b^{i} \wedge\left(f_{i}{ }^{j} * \vartheta\right) \wedge i_{t_{j}}\left(\vartheta^{\prime}\right)$, where $\vartheta, \vartheta^{\prime}$ are generic forms and $V=b^{i} i_{t_{i}}$.

Proof.

Properties 1), 2), 3) and 4) follow directly from the definition (6.4).

Property 5) is also a consequence of definition (6.4); the proof makes use of (6.1) and $d\left(f_{j}{ }^{k} * \vartheta\right)=f_{j}{ }^{k} * d \vartheta$.

\section{Commutation relations}

The careful choice of conventions (see introduction) allows that all relations can be summarized as deformed commutation relations 9 of right-acting operators $(\vartheta$ : $p$-form, $\left.\quad V=b^{i} t_{i}\right)$

$$
\begin{aligned}
d \vartheta & =d(\vartheta)+(-1)^{p} \vartheta d \\
i_{V} \vartheta & =i_{V}(\vartheta)+(-1)^{p} b^{i}\left(f_{i}{ }^{j} * \vartheta\right) i_{t_{j}} \\
\ell_{V} \vartheta & =\ell_{V}(\vartheta)+b^{i}\left(f_{i}^{j} * \vartheta\right) \ell_{t_{j}}+(-1)^{p} d b^{i}\left(f_{i}{ }^{j} * \vartheta\right) i_{t_{j}}
\end{aligned}
$$


and their actions ( $a \in A$, rest as above) as given by:

$$
i_{V}(a)=0 ; \quad i_{V}\left(\omega^{j}\right)=b^{j} ; \quad \ell_{V}(a)=V(a)=b^{i} \chi_{i} * a ; \quad \ell_{V} \omega^{j}=b^{i} \chi_{i} * \omega^{j}+d b^{j} .
$$

These operators form a graded quantum Lie algebra

$$
\begin{aligned}
\{d, d\} & =0 \\
{\left[d, \ell_{V}\right] } & =0 \\
\left\{d, i_{V}\right\} & =\ell_{V}
\end{aligned}
$$

which is supplemented by two more relations]

$$
\begin{aligned}
& {\left[\ell_{t_{i}}, \ell_{t_{k}}\right]_{\hat{B}}=\ell_{\left[t_{i}, t_{k}\right]_{\hat{B}}}=f_{i k}{ }^{l} \ell_{t_{l}}} \\
& {\left[\ell_{t_{i}}, i_{t_{k}}\right]_{\hat{B}}=i_{\left[t_{i}, t_{k}\right]_{\hat{B}}}=f_{i k}{ }^{l} i_{t_{l}}}
\end{aligned}
$$

where $\hat{B}_{i k}^{r s} \equiv\left(\widehat{\sigma^{-1}}\right)_{i k}^{r s}=f_{i}{ }^{s}\left(N^{r}{ }_{k}\right),\left[\ell_{t_{i}}, \ell_{t_{k}}\right]_{\hat{B}} \equiv \ell_{t_{i}} \ell_{t_{k}}-\hat{B}_{i k}^{r s} \ell_{t_{r}} \ell_{t_{s}}$ and $f_{i k}^{l}=\chi_{i}\left(N_{k}^{l}\right)$.

Using the right product (4.12) the commutation relations can be restated in basis-independent form as

$$
V \vartheta=V(\vartheta)+V_{\square \vartheta} .
$$

Here and in the following $V$ stands for any of the first-order operators $V=b^{i} t_{i}$ (for $\vartheta \in A$ only) or $i_{V}, \ell_{t_{i}}$ and $d$. For example:

$$
t_{i} a=t_{i}(a)+t_{i} a=\chi * a+\left(f_{i}^{j} * a\right) t_{j} ; \quad d \vartheta=d(\vartheta)+d_{\square} \vartheta=d(\vartheta)+(-1)^{\operatorname{deg}(\vartheta)} \vartheta d .
$$

For $\vartheta$ a $p$-form one must add the following rules to the definition (4.12) of the right product to take care of the grading:

$$
d \square \vartheta=(-1)^{p} \vartheta d ; \quad i_{V} \vartheta=(-1)^{p} b^{i}\left(f_{i}^{j} * \vartheta\right) i_{t_{j}} .
$$

Note that $V$ appears twice in the r.h.s. of $(7.14)$. These are the two parts of the (classical) coproduct

$$
\delta(V)=V \otimes 1+1 \otimes V .
$$

In a hopefully self-explanatory notation we rewrite (7.14) as

$$
V \vartheta=\delta(V) \square \vartheta
$$

so that the role of $\delta(V)$ is emphasized.

We now extend the definition of $\delta$ to composition of operators, say $V \cdot V^{\prime} \cdots V^{\prime \prime}$ :

$$
\forall \vartheta \in \Gamma^{\wedge} \quad \delta\left(V \cdot V^{\prime} \cdots V^{\prime \prime}\right) \square \vartheta \equiv V \cdot V^{\prime} \cdots V^{\prime \prime} \vartheta .
$$

\footnotetext{
${ }^{7}$ Relations for the inner derivations can be constructed along the lines of section 5.1 .

${ }^{8}$ In these notations the Cartan-Maurer formula reads:

$d \omega^{i} \otimes \chi_{i}=\omega^{i} \otimes \omega^{j} \otimes \chi_{j} \chi_{i}=-\omega^{a} \wedge \omega^{b} \hat{\sigma}_{a b}^{p q}\left[\chi_{q}, \chi_{p}\right]_{\hat{B}}$. In particular $d \omega^{i}=\omega^{a} \otimes \omega^{b} \chi_{a}\left(M_{b}{ }^{i}\right)$.
} 
From equation (2.33) one can prove that

$$
f_{i}^{k} *\left[i_{t_{j}}(\vartheta)\right]=\hat{B}_{i j}^{r s} i_{t_{r}}\left[f_{s}^{k} * \vartheta\right]
$$

the following theorem is then easily derived:

\section{Theorem 8.}

The operators $i_{V}, \ell_{t_{i}}, d$ and $V\left(V=b^{i} t_{i}\right.$ is only defined on $\left.A\right)$ form a braided tensor algebra. The braidings are given by:

$$
\begin{gathered}
\left(1 \otimes a^{i} i_{t_{i}}\right)\left(b^{j} i_{t_{j}} \otimes 1\right)=-a^{i}\left(f_{i}{ }^{h} * b^{j}\right) \hat{B}_{h j}^{r s}\left(i_{t_{r}} \otimes i_{t_{s}}\right) ; \quad(1 \otimes d)(d \otimes 1)=-d \otimes d \\
(1 \otimes d)\left(i_{V} \otimes 1\right)=-i_{V} \otimes d ; \quad\left(1 \otimes i_{V}\right)(d \otimes 1)=-d \otimes i_{V}
\end{gathered}
$$

Using (6.3) we also get all the other braidings, e.g.

$$
\left(1 \otimes a^{i} \ell_{t_{i}}\right)\left(b^{j} \ell_{t_{j}} \otimes 1\right)=a^{i}\left(f_{i}^{h} * b^{j}\right) \hat{B}_{h j}^{r s}\left(\ell_{t_{r}} \otimes \ell_{t_{s}}\right) .
$$

From definition (7.18) and the above theorem it follows:

\section{Theorem 9.}

$\delta$ is a homomorphism in the braided tensor algebra of operators.

For example:

$$
\delta\left(t_{i} t_{j}\right)=\delta\left(t_{i}\right) \delta\left(t_{j}\right)
$$

indeed

$$
\begin{aligned}
\delta\left(t_{i} t_{j}\right) \triangleright a & \equiv t_{i} t_{j} a=t_{i} t_{j}(a)+t_{i}\left(f_{j}^{l} * a\right) t_{l} \\
& =t_{i}\left(t_{j}(a)\right)+\left(f_{i}^{l} * t_{j}(a)\right) t_{l}+\left[t_{i}\left(f_{j}^{l} * a\right)\right] t_{l}+\left(f_{i}^{m} * f_{j}{ }^{l} * a\right) t_{m} t_{l} \\
& =\left(t_{i} t_{j} \otimes 1+(1+\hat{B})_{i j}^{r s}\left(t_{r} \otimes t_{s}\right)+1 \otimes t_{i} t_{j}\right) \triangleright a \\
& =\left(t_{i} \otimes 1+1 \otimes t_{i}\right)\left(t_{j} \otimes 1+1 \otimes t_{j}\right) \square a \\
& =\left[\delta\left(t_{i}\right) \delta\left(t_{j}\right)\right]_{\square} a
\end{aligned}
$$

where we have used $\hat{B}_{i j}^{r s} \chi_{r} * f_{s}{ }^{l}=f_{i}{ }^{l} * \chi_{j}$ an identity easily derived from (6.3) and (7.19), or directly from $d\left(f_{i}{ }^{j} * a\right)=f_{i}{ }^{j} * d a$.

As another example we have:

$$
\delta\left(\ell_{V}\right)=\delta i_{V} \delta d+\delta d \delta i_{V}
$$

indeed use the braiding (7.20) to show that $\left(\delta i_{V} \delta d+\delta d \delta i_{V}\right) \bullet \vartheta$ is given by (7.7).

To summarize: we can state commutation relations for a general differential operator $D$ and for any form $\vartheta$ in an abstract way as

$$
D \vartheta=\delta(D) \square \vartheta
$$

where $\delta$ is the classical coproduct on first order operators and can be extended (Theorem 9) as a homomorphism to higher order operators. 


\section{A second Lie derivative and a puzzle}

It is natural to define a Lie derivative $\ell_{h_{i}}^{\mathcal{R}}$ of a generic form $\tau$ along a right-invariant vector field $h_{i}$ in terms of the left coaction $\Delta_{\Gamma}$ :

$$
\ell_{h}^{\mathcal{R}}(\tau) \equiv(\chi \otimes i d) \Delta_{\Gamma}(\tau)=\tau * \chi
$$

just like it was natural that we used the right coaction, when we defined $\ell_{t_{i}}$ in the previous section. In this section we would like to compare the two definitions.

From the above definition we find

$$
\begin{aligned}
\ell_{h_{i}}^{\mathcal{R}}\left(\vartheta \vartheta^{\prime}\right) & =\chi\left(\vartheta_{1} \vartheta^{\prime}{ }_{1}\right) \vartheta_{l} \vartheta^{\prime}{ }_{l} \\
& =\chi\left(\vartheta_{1}\right) \vartheta_{l} \vartheta^{\prime}+f_{i}{ }^{j}\left(\vartheta_{1}\right) \chi_{j}\left(\vartheta^{\prime}{ }_{1}\right) \vartheta_{l} \vartheta^{\prime}{ }_{l} \\
& =\ell_{h_{i}}^{\mathcal{R}}(\vartheta) \vartheta^{\prime}+\left(\vartheta * f_{i}{ }^{j}\right) \ell_{h_{j}}^{\mathcal{R}}\left(\vartheta^{\prime}\right)
\end{aligned}
$$

and, in a particular case:

$$
\begin{aligned}
\ell_{h_{i}}^{\mathcal{R}}(d a b) & =d\left(h_{i}(a)\right) b+\left(d a * f_{i}{ }^{j}\right) h_{j}(b) \\
& =d\left(h_{i}(a)\right) b+\left(d a * f_{i}{ }^{j}\right) S^{-1} N^{k}{ }_{j} t_{k}(b) .
\end{aligned}
$$

On the other hand we can use the results of the previous chapter to give an alternative expression for the Lie derivative along the right-invariant vector field $h_{i}$ :

$$
\begin{aligned}
\ell_{h_{i}} & =S^{-1} N^{j}{ }_{i} \ell_{t_{j}}+d\left(S^{-1} N^{j}{ }_{i}\right) \wedge i_{t_{j}} \\
& =M_{i}{ }^{j} \ell_{t_{j}}+d M_{i}{ }^{j} \wedge i_{t_{j}}
\end{aligned}
$$

and hence

$$
\begin{aligned}
\ell_{h_{i}}(d a b) & =d\left(M_{i}{ }^{j} t_{j}(a)\right) b+M_{i}{ }^{j}\left(f_{j}{ }^{k} * d a\right) t_{k}(b) \\
& =d\left(h_{i}(a)\right) b+M_{i}{ }^{j}\left(f_{j}{ }^{k} * d a\right) t_{k}(b) .
\end{aligned}
$$

The difference between expressions (8.26) and (8.28) is a good index for the "defect" between left and right transports on a quantum group:

$$
\begin{aligned}
\left(\ell_{h_{i}}-\ell_{h_{i}}^{\mathcal{R}}\right)(d a b) & =\left[M_{i}{ }^{j}\left(f_{j}{ }^{k} * d a\right)-\left(d a * f_{i}{ }^{j}\right) M_{j}{ }^{k}\right] t_{k}(b) \\
& =-D I_{i}{ }^{k}(a) t_{k}(b) ; \\
D I_{i}{ }^{k}(a) \equiv\left[d\left(M_{i}{ }^{j}\right)\left(f_{j}{ }^{k} * a\right)\right. & \left.-\left(a * f_{i}{ }^{j}\right) d\left(M_{j}{ }^{k}\right)\right] \quad \text { (Defect Index). }
\end{aligned}
$$

In the last passage we have used the Leibniz rule for $d$ combined with (2.33). The term in the square brackets is always zero in the classical (undeformed) case. Note that $\left(\ell_{h_{i}}-\ell_{h_{i}}^{\mathcal{R}}\right)$ vanishes on $d a$ and $b$ separately but not necessarily on $d a b$. The case of " $b$ " confirms a result from Section 4 :

$$
\ell_{h_{i}}(b)=S^{-1} N^{j}{ }_{i} t_{j}(b)=h_{i}(b)=\ell_{h_{i}}^{\mathcal{R}}(b)
$$


and shows that we will not encounter any ambiguities or inconsistencies as long as we deal with general vector fields and functions alone. Problems can occur however when we start to introduce forms. Here is what happens in a well known example:

In the approach [3, 13] to differential calculus on quantum groups that is based on the $L^{ \pm}$and $T$ matrices of [1] one can give the following explicit expressions for the $f_{i}{ }^{j}$ and the linearly independent $\chi_{k}$ functionals (using double-indices)

$$
f_{i_{1} i_{2}}^{j_{1} j_{2}}=L^{+i_{1}}{ }_{j_{1}} S L^{-j_{2}}{ }_{i_{2}}=\varepsilon \delta_{k_{2}}^{k_{1}}-L^{+k_{1}} S L^{-j}{ }_{k_{2}}
$$

and for the adjoint representation $N^{j}{ }_{i}, M_{i}{ }^{j}$

$$
N^{j_{1} j_{2}}{ }_{i_{1} i_{2}}=S T^{i_{1}}{ }_{j_{1}} T^{j_{2}}{ }_{i_{2}} \quad, \quad M_{i_{1} i_{2}}{ }^{j_{1} j_{2}}=\left(S^{-1} T^{j_{2}}{ }_{i_{2}}\right) T^{i_{1}}{ }_{j_{1}} .
$$

We want to investigate whether the "Defect Index" vanishes. First consider the $x^{a_{1}} a_{2}$ of (2.4) such that $\chi_{{ }_{k_{2}}}^{k_{1}}\left(x^{a_{1}}{ }_{a_{2}}\right)=\delta^{k_{1} a_{1}} \delta_{k_{2} a_{2}}$ and notice that $t^{k_{1}}{ }_{k_{2}}\left(x_{2 a}^{a}\right) S^{-1}\left(x_{1 a}^{a}\right)=\delta_{k_{2}}^{k_{1}}$ Therefore

$$
\left(\ell_{h_{i}}-\ell_{h_{i}}^{\mathcal{R}}\right)\left(d a x_{2 a}^{a}\right) S^{-1}\left(x_{1 a}^{a}\right)=\sum_{k_{1}=k_{2}} D I_{i}^{k}(a)=\sum_{k_{1}=k_{2}} d\left(M_{i}^{j}\right)\left(f_{j}{ }^{k} * a\right)-\left(a * f_{i}^{j}\right) d\left(M_{j}{ }^{k}\right) .
$$

Now $f_{j_{1} j_{2}}{ }^{k k}$ gives a non-trivial matrix $Y^{j_{1}}{ }_{j_{2}}$, while $M_{j_{1} j_{2}}{ }^{k k}$ will become the Kronecker symbol $I \delta^{j_{1}}{ }_{j_{2}}$ which vanishes as it is acted upon by $d$. This is what remains if the Defect Index were zero:

$$
d\left(M_{i}^{j}\right)\left(Y_{j} * a\right)=0
$$

for all $a \in A-$ an incorrect statement!

\section{Discussion}

We have seen that the bicovariant differential calculus studied so far admits a natural contraction operator along generic vector fields which leads to a Lie derivative $\ell$ that is not unique. We stress that there are no problems when we deal with vector fields and functions only. This should suffice in many physics applications (e.g. gauge theories) that do not make direct reference to volume forms. To evaluate the defect index we have used the Leibniz rule for the exterior differential $d$ [see (8.27) and (8.28)], this shows that a possible way to obtain a differential calculus with $\ell=\ell^{\mathcal{R}}$ is to define a new exterior differential $d$ that satisfies a deformed Leibniz rule [17]. The problem of the non-unique Lie derivative adds to another mystery [18 of differential calculi on quantum groups: the number of forms is generally larger than one should expect from the classical limit. Notice also that the Defect Index is strictly connected with the impossibility of the Lie derivative to be a good differential operator i.e. satisfying a deformed Leibniz rule. Indeed, consider $V_{k} \equiv M_{k}{ }^{j} t_{j}$; since $V_{k}$ has a tensor index we would like $\ell_{V_{k}}$ to exhibit a nice (braided) 
deformation of Leibniz rule. Using 5) of Theorem 7 we find

$$
\begin{aligned}
\ell_{V_{i}}\left(a \vartheta^{\prime}\right) & =\left(\ell_{V_{i}} a\right) \vartheta^{\prime}+\left(a * f_{i}^{j}\right) \ell_{V_{j}} \vartheta^{\prime}+\left[d M_{i}^{j} f_{j}{ }^{k} * a-a * f_{i}^{j} d M_{j}{ }^{k}\right] \wedge i_{t_{k}}\left(\vartheta^{\prime}\right) \\
& =\left(\ell_{V_{i}} a\right) \vartheta^{\prime}+\left(a * f_{i}{ }^{j}\right) \ell_{V_{j}} \vartheta^{\prime}+D I_{i}{ }^{k}(a) \wedge i_{t_{k}}\left(\vartheta^{\prime}\right) .
\end{aligned}
$$

It is the Defect Index that is responsible for the appearance of the contraction operator in the Leibniz rule for the Lie derivative.

\section{References}

[1] L.D. Faddeev, N.Yu. Reshetikhin, L.A. Takhtajan, Quantization of Lie Groups and Lie Algebras, Algebra i Anal. 11 (1989) 178 (Leningrad Math. J. 1193 (1990)).

[2] S.L. Woronowicz, Differential Calculus on Compact Matrix Pseudogroups, Commun. Math. Phys. 122 (1989) 125.

[3] B. Jurčo, Differential calculus on quantized simple Lie groups, Lett. Math. Phys. 22 (1991) 177.

[4] U. Carow-Watamura, M. Schlieker, S. Watamura, W. Weich, Bicovariant differential calculus on quantum groups $S U_{q}(N)$ and $S O_{q}(N)$, Commun. Math. Phys. 142 (1991) 605.

[5] L. Castellani,Differential calculus on $I_{S O}(N)$, quantum Poincaré algebra and q-gravity, DFTT-70/93, hep-th 9312179, to be publ. in Commun. Math. Phys.; The Lagrangian of q-Poincaré Gravity, Phys. Lett. B327 (1994) 22.

[6] P. Schupp, P. Watts and B. Zumino, Differential Geometry on Linear Quantum Groups, Lett. Math. Phys. 25 (1992) 139.

[7] P. Aschieri and L. Castellani, An introduction to non-commutative differential geometry on quantum groups, Int. J. Mod. Phys. A8 (1993) 1667.

[8] P. Schupp, P. Watts and B. Zumino, Bicovariant Quantum Algebras and Quantum Lie Algebras, Commun. Math. Phys. 157 (1993) 305.

[9] P. Schupp, P. Watts, B. Zumino, Cartan Calculus on Quantum Lie Algebras, XXII $^{\text {th }}$ DGM, Ixtapa, 1993; Preprint LBL-34833 (1993); hep-th/9312073.

[10] P. Schupp, P. Watts, Universal and generalized Cartan calculus on Hopf algebras, Preprint LBL-33655 (1994); hep-th/9402134.

[11] M.E. Sweedler, Hopf Algebras, (Benjamin, New York, 1969).

[12] P. Aschieri, The Space of Vector Fields on Quantum Groups, preprint UCLA/93/TEP/25 (1993); hep-th/9311151. 
[13] B. Zumino, Introduction to the differential Geometry of Quantum Groups, in: K. Schmüdgen (ed.), Math. Phys. X, Proc. X-th IAMP Conf. Leipzig (1991), Springer-Verlag (1992).

[14] P. Schupp, Quantum Groups, Non-Commutative Differential Geometry and Applications, PhD. thesis, Berkeley (1993); preprint LBL-34942; hep-th/9312075.

[15] P. Schupp, Cartan Calculus: Differential Geometry for Quantum Groups, Enrico Fermi Summer School on Quantum Groups, Varenna (1994); preprint LMU-TPW-94-8; hep-th/9408170.

[16] A. Sudbery, Canonical Differential Calculus on Quantum General Linear Groups and Supergroups, Phys. Lett. B284 (1992) 61, erratum -ibid. B291 (1992) 519 .

[17] L.D. Faddeev, P.N. Pyatov, The Differential Calculus on Quantum Linear Groups; hep-th/9402070

[18] A. Sudbery, The Quantum Orthogonal Mystery, $30^{t} h$ Karpacz Winter School on Theoretical Physics: Quantum Groups, Poland (1994); hep-th/9407110 